\title{
Information on the Causal Relationship Between Store kaizen and Store Features That Attract Customers by Covariance Structural Analysis
}

\author{
Yumiko Taguchi ${ }^{1}$, Yasushi Akiyama ${ }^{2}$, and Tsutomu Tabe ${ }^{3}$ \\ ${ }^{1}$ Department of Business Administration and Communication, Shohoku College, 428 \\ Nurumizu, Atsugi City, Kanagawa 243-8501 Japan \\ taguchi@shohoku.ac.jp \\ ${ }^{2}$ Hewlett-Packard Japan, Ltd., System Integrator Sales, 7, Gobancho, Chiyoda-ku, Tokyo, \\ 102-0076 Japan \\ yasushi.akiyama@hp.com \\ ${ }^{3}$ Department of Industrial and Systems, Engineering College of Science and Engineering, \\ Aoyama Gakuin University 5-10-1 Fuchinobe, Sagamihara City, Kanagawa 229-8558 Japan \\ tabe@ise.aoyama.ac.jp
}

\begin{abstract}
This research has two objectives: first, to develop a method to quantitatively clarify the causal relationship between store improvements (store kaizen) and store features that attract customers; second, to verify the effectiveness of the method developed. The results of this research confirm two points. (1) The causal relationship was quantitatively clarified through the use of a covariance structural analysis, and the degree of the relationship between the store features that attract customers and store kaizen could be understood. (2) The proposed methodology has proven to be useful in an actual store improvement activity. The owner of the store studied in the investigation commented, "It is very important to set up a hypothesis when seeking an improvement. This research provides a helpful framework for doing so."
\end{abstract}

Keywords: store features that attract customers, store kaizen, causal relationship, covariance structural analysis.

\section{Introduction}

The sales of small and medium-sized supermarket chains in Japan are hovering at low levels due to the changes of consumer needs and fiercer competition ${ }^{1}$. If the owner of a small and medium-sized supermarket chain in Tokyo wishes to make store improvements (referred to as "store kaizen" hereafter) he can take the following steps: 1) grasp the current problems, based on periodic surveys of customer satisfaction in his own stores and the stores of competitors; 2) consider management adjustments and

${ }^{1}$ According to a survey of commercial statistics by the Ministry of Economy, Trade and Industry, the growth rate of annual sales in "food stores" is decreasing (surveyed in 1994, 1997, 2002). 
improvements for his stores, and implement those that are feasible [1]. With this approach, however, it is difficult for the owner to determine the effects of kaizen on sales (improvements of the extracted problems). Thus, it is difficult for the owner to accurately grasp which problems should be improved.

Customers generally want to shop at stores with stable sales and abundant merchandise and services that the customers seek. In this research we think of these as stores with features that attract customers. To increase sales, it is generally thought that the owners must improve their stores (kaizen) by adding more store features that attract customers. In former research we developed several information systems targeting store kaizen [1][2]. These systems have drawbacks, however, as they fail to provide information concrete enough to allow the owners to: 1) grasp how the extracted problems influence the store features that attract customers, and 2) carry out the kaizen corresponding to the problems. If more concrete information on the causal relationship between store features that attract customers and the problems to be improved is provided to the owner, the owner can rapidly make improvements and maintain and enhance the features that attract customers in his store. Thus, we seek to develop a method capable of providing this type of information. This research aims to accomplish two tasks as means to facilitate the efforts of store owners to carry out store kaizen by maintaining and enhancing the store features that attract customers: first, to develop a method to clarify the causal relationship between store features that attract customers and store kaizen; second, to verify the effectiveness of the method developed.

\section{Approach to Solve Problems}

This chapter describes a basic strategy for grasping the causal relationship between the store features that attract customers, the problems to be improved, and the method to realize the above strategy.

\subsection{Basic Strategy}

This research focuses on the two following points.

- Quantifying and visualizing the causal relationship between store kaizen and the store features that attract customers

To carry out store kaizen as a means of effectively maintaining and enhancing the store features that attract customers, it is helpful to quantify and visualize the relationship between the store features that attract customers and store kaizen.

- Taking up the causal relationship which has a construct linked to "store features that attract customers."

This research proposes a method for clarifying the causal relationship, with mechanisms built in to consider concepts that cannot be directly measured by questionnaires. 


\subsection{Considering the Method}

Regression analysis can be used as a representative method to quantify causal relationships. The method can capture a causal relationship between measured values, but not a causal relationship that includes abstract concepts that defy measurement. Factor analysis can explore common factors (referred to as "factors" hereafter) behind several variables, but cannot capture a causal relationship of factors and variables. Fortunately we can use a third alternative, covariance structural analysis [3], to compensate for the faults of regression and factor analyses in the handling of the same task. This research applies covariance structural analysis to clarify a causal relationship that includes abstract concepts.

\section{Applying Covariance Structural Analysis}

This chapter applies covariance structural analysis in order to clarify the causal relationship between store kaizen and store features that attract customers. We describe a tentative hypothesis, observed and latent variables, indexes to evaluate a model representing the hypothesis, and a method for developing such a model to apply covariance structural analysis.

\subsection{Tentative Hypothesis}

Prior research [1][2] has clarified that consumers base their impressions of shops on the merchandise, the counters, the services offered, etc. If store kaizen is performed based on the degree of customer satisfaction with each item, the store features that attract customers can be maintained and raised. Interviews of shop owners and large groups of housewives elicited comments such as the following: "The quality of the service, whether it's good or bad, forms my impression of the store"; "The cleanliness of the counter changes my impression of the quality of the merchandise." This informs us that the three factors of "service," "counter," and "merchandise," influence other factors. Conceivably, they may influence other factors that determine a store's success in enhancing store features that attract customers.

On this basis, we formed the following tentative hypothesis: "The services, the counter and the merchandise in the store are closely related to each other, hence they influence the store features that attract customers." After forming this tentative hypotheses, we verified it by the following methodology: 1) asking the owner to make improvements in their (store kaizen), 2) developing two models of covariance structural analysis, i.e., a model before store kaizen and a model after store kaizen, 3) comparing the two models.

\subsection{Variables}

Three types of variables, i.e., latent variables, observed variables, and error variables, are used to develop the models in the covariance structural analysis. 


\subsubsection{Latent Variables}

There are two types of latent variables. The first is "store features that attract customers." The second is latent variables for the constructs if the constructs behind the observed variables can be found. The use of the latent variables allows us to arrange the observed variables and easily interpret the model.

\subsubsection{Observed Variables}

There are also two types of observed variables, as shown in table 1. First are variables for measuring the customers' evaluations of each factor targeted as store kaizen. Second are the variables for measuring the store features that attract customers. The factors include items i.e., merchandise, service, counters, and the total impression of the store, based on tentative hypotheses. Variables X1 to X12 are the degrees of customer satisfaction with three items, i.e., merchandise, service, and counters. Variable X13 is the degree of total customer satisfaction with the store.

Moreover, three variables are used to measure the store features that attract customers: 1) a variable indicating how frequently the customer visits the store, 2) a variable indicating how many times the customer has visited previously, and 3) a variable indicating the degree of the customer's intention to visit again, based on the definition of the store features that attract customers in Chapter 1. A questionnaire with a five-grade evaluation format was used to collect data in relation to these variables.

\subsubsection{Error Variables}

If the error variables have a large influence in the covariance structural analysis, the observed variable will not be useful for explaining the relation of the model. Therefore, we set up error variables for all of the observed variables.

\subsection{Developing the Model}

We verify the above by considering the alternative models before and after store kaizen, respectively. The flow of developing models has six steps.

- Step 1: collecting data on observed variables by conducting a customer survey in front of the store.

- Step 2: considering whether there are factors behind the observed variables (that is to say, constructs) in the customer evaluations of each factor that can be targeted as store kaizen, as shown in table 1, by conducing an exploratory factor analysis (hereafter described as "factor analysis") based on the response data in gained in step 1.

- Step 3: depicting one of the alternative models by a path diagram.

- Step 4: setting response data in the path diagram.

- Step 5: performing a covariance structural analysis.

- Step 6: repeating step 3 to step 5, as above, on other (alternative) models.

To consider the models effectively based on the flow, we use statistical software such as SPSS for factor analysis, and AMOS for covariance structural analysis. 
Table 1. List of observed variables

\begin{tabular}{|c|c|}
\hline Types & Variable : meaning \\
\hline $\begin{array}{l}\text { Customer evaluations of } \\
\text { each factor which could be } \\
\text { targeted as store kaizen }\end{array}$ & $\begin{array}{l}\text { X1 : degree of satisfaction with the quality of vegetables } \\
\text { X2 : degree of satisfaction with the assortment of vegetables } \\
\text { X3 : degree of satisfaction with the quality of the fish } \\
\text { X } 4: \text { degree of satisfaction with the assortment of the fish } \\
\text { X } 5: \text { degree of satisfaction with the quality of the meat } \\
\text { X6 : degree of satisfaction with the assortment of the meat } \\
\text { X7 : degree of satisfaction with the quality of the delicatessen } \\
\text { X8 : degree of satisfaction with the assortment of the delicatessen } \\
\text { X } 9: \text { degree of satisfaction with the quality of other food } \\
\text { X10 : degree of satisfaction with the assortment of other food } \\
\text { X11: degree of satisfaction with the staff's attitude toward } \\
\text { customers } \\
\text { X12: degree of satisfaction with the cleanliness } \\
\text { X13 : degree of customer satisfaction with the store overall }\end{array}$ \\
\hline $\begin{array}{l}\text { Store features that attract } \\
\text { customers }\end{array}$ & $\begin{array}{l}\text { X14 : how frequently customers visit the store } \\
\text { X15 : how many times customers have visited the store } \\
\text { X16: the degree of customer intention to visit the store again }\end{array}$ \\
\hline
\end{tabular}

\subsection{Evaluating the Models}

One way to evaluate and select a final model from among a set of alternative models is to remove the models with a GFI (Goodness of Fit Index) of under 0.9 and adopt the model with the smallest AIC (Akaike's Information Criterion) from among those remaining. This research develops several alternative models by covariance structural analysis, then consider the models using GFI and AIC. We also consider the relationships between variables based on test statistics, then modify the models based on our consideration.

\section{Developing a Model for a Real (Bricks-and-Mortar) Store}

This chapter describes the development of two models, one before store kaizen and one after store kaizen, based on the targeted real store as mentioned in chapter 3 , and examines each.

\subsection{Model Before Kaizen}

When developing several models of the store before kaizen, the three following models had GFIs of over 0.9 .

- I. A model referenced based on the results of factor analysis, including latent variables.

- II. A model referenced based on advice from the store owner.

- III. A model encompassing paths directly drawing "store features that attract customers" and the "customer evaluations of each factor which could be targeted for store kaizen." 
When executing covariance structural analysis based on the three models, the GFIs of all models are over 0.9 . Thus, all models are considered to have good explanatory ability. The AICs of all models are shown in table 2. Model I has the smallest AIC, as shown in fig. 1. Therefore, we adopted model I as our model before kaizen.

Table 2. The values of AIC on alternative models before store kaizen

\begin{tabular}{|c|c|c|c|}
\hline Model & I & II & III \\
\hline AIC & 93.13 & 96.58 & 106.50 \\
\hline
\end{tabular}

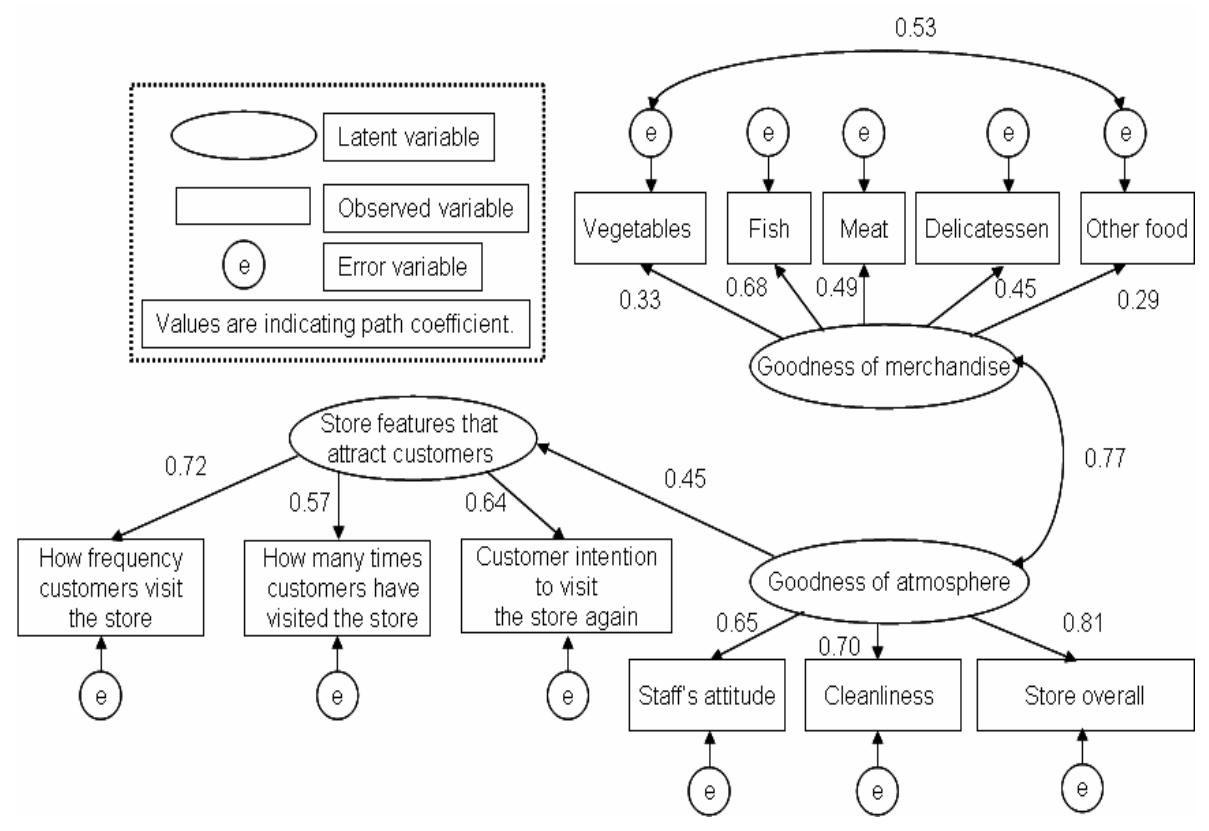

Fig. 1. Result of analysis in the model I

\subsection{Model After Kaizen}

Once we completed the store kaizen focused on merchandise, service, and counters, we developed alternative models of the store after kaizen. The six following models had GFIs of over 0.9 .

- i. A model referenced based on the results of factor analysis, including latent variables.

- ii: A model which grouped the variables related to "quality" (i.e., X1) and the variables related to "assortment" (i.e., X2) together as one latent variable (i.e., goodness of vegetables) for each article (i.e., vegetables).

- iii: A model which grouped the variables related to "quality" (i.e., X1) and the variables related to "assortment" (i.e., X2) together as one latent variable (i.e., goodness of vegetables) for each article (i.e., vegetables) and then grouped them as one latent variable (i.e., goodness of merchandise). 
- iv: A model referenced based on advice by the store owner based on model i above.

- v: referenced based on advice by the store owner based on model iii above.

- vi: A model encompassing paths directly drawing "store features that attract customers" and the "customer evaluations of each factor which could be targeted for store kaizen."

When executing covariance structural analysis based on the six models, the values of GFI in all models are over 0.9. Therefore, all models are considered to have good explanatory ability. The AICs of all models are shown in table 3. Model iii has the smallest AIC, as shown fig. 2. Therefore, we adopted model iii as the model after kaizen.

Table 3. AICs in alternative models after kaizen

\begin{tabular}{|c|c|c|c|c|c|c|}
\hline Model & i & ii & iii & iv & v & vi \\
\hline AIC & 176.80 & 172.79 & 166.13 & 178.38 & 166.93 & 211.19 \\
\hline
\end{tabular}

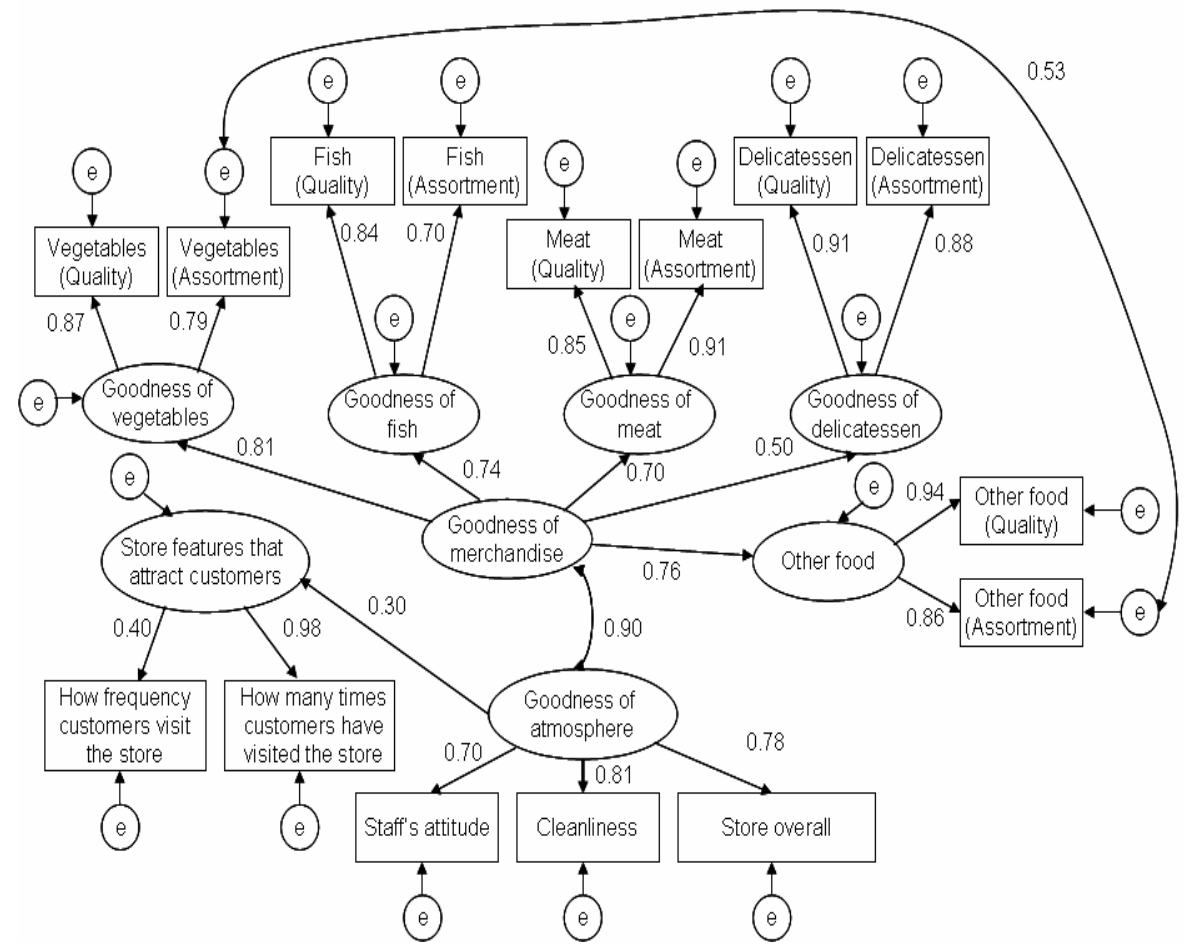

Fig. 2. Analytical results for model iii 


\subsection{Examining the Models}

This section considers the relationship between the store features that attract customers and the store kaizen based on the results of analyses adopted in 4.1 and 4.2.

\subsubsection{Model Before Kaizen}

In the model before store kaizen, enhancement in "goodness of atmosphere" is ultimately linked to enhancement in "the store features that attract customers." Factors that can be targeted as store kaizen, such as merchandise, service, and counters, do not affect "the store features that attract customers." On the other hand, they can influence "the store features that attract customers" through "goodness of atmosphere." In addition, the features of this model can be understood based on three path coefficients (see fig. 1): 1) path "goodness of atmosphere" and "store features that attract customers," 2) path "goodness of merchandise" and "goodness of atmosphere," and 3) "goodness of merchandise" and "Fish."

In 1) mentioned above, an enhancement in "goodness of atmosphere" reflects enhancements in "the degree of satisfaction with the staff's attitude toward customers," "the degree of satisfaction with cleanliness," and "the degree of customer satisfaction with the store overall." By inference, therefore, these variables can be assumed to influence "the store features that attract customers." In 2) above, an enhancement in "goodness of merchandise" can be inferred to reflect an enhancement in the "goodness of atmosphere." Further, the "goodness of merchandise" influences_"the store features that attract customers" without directly influencing them, as consumers take it for granted that the merchandise at a supermarket should be good. Moreover, we can infer that a store's efforts take better care of its customers and improve in-store hygiene will enhance "the store features that attract customers" more than they enhance degree of satisfaction with the merchandise. In 3) above, "fish" is most robustly influenced by the "goodness of merchandise," as the targeted store was initially barraged by requests for fish and subsequently faced only a low demand for fish. Therefore, we can infer that an enhancement in the degree of satisfaction with fish is linked to "goodness of merchandise."

\subsubsection{Model After Store Kaizen}

In the model after store kaizen, enhancement in "goodness of atmosphere" is ultimately linked to enhancement in "the store features that attract customers." Factors that can be targeted as store kaizen do not directly influence "the store features that attract customers." On the other hand, they can influence "the store features that attract customers" through "goodness of atmosphere." In addition, the features of this model can be understood based on three path coefficients (see fig. 2): 1) path "goodness of atmosphere" and "the store features that attract customers," 2) path "goodness of merchandise" and "goodness of vegetables" and 3) "goodness of merchandise" and "goodness of delicatessen."

In 1) mentioned above, an enhancement in "goodness of atmosphere" reflects enhancements in "the degree of satisfaction with the staff's attitude toward customers," "the degree of satisfaction with the cleanliness of the store," and "the degree of customer satisfaction with the store overall." By inference, therefore, these variables can be assumed to influence "the store features that attract customers." Further, the 
"goodness of merchandise" influences "the store features that attract customers" without directly influencing them. In 2) above, the "goodness of vegetables" is most robustly influenced by the "goodness of merchandise." This could be explained by the soaring price of vegetables during the heavy snows at the end of 2005, when the customer survey was conducted. Vegetables were in short supply, hence we can infer that the degree of customer satisfaction dropped. In 3) above, the "goodness of delicatessen" is most weakly influenced by the "goodness of merchandise," due to relative abundance of New Year delicacies in the delicatessen at the end of the year, when the customer survey was executed. Further, we can infer that the store kaizen to "delicatessen" executed does not influence "the store features that attract customers."

\section{Validation}

In this chapter we examine whether the method to clarify the causal relationship between the store kaizen and the "store features that attract customers" is useful. To consider this, we ask the store owner two questions. First, we ask if the method developed clarifies the causal relationship between "store features that attract customers" and the factors which can be targeted as store kaizen. Second, we ask if the method is helpful for the store kaizen of a real store.

\subsection{Method for Clarifying the Causal Relationship Between Store Features That Attract Customers and Factors Relative to Store Kaizen}

When we asked the store owner to judge the result of the examinations based on the two analyses of the models (the models before and after kaizen) at the bricks-and-mortar store described in the last chapter, he responded as follows: "It was more interesting for me to generate new ideas for store kaizen with this method, compared with the methods used in your former research."

\subsection{Helpfulness of the Method for the Store Kaizen of the Bricks-and-Mortar Store}

When we asked the owner if the method was helpful for the store kaizen of the bricks-and-mortar store, he responded with two comments:

"It is very important to set up a hypothesis when seeking an improvement. This research provides a helpful framework for doing so."

"To enhance 'the store features that attract customers,' I can prioritize the kaizen by grasping the factors influence directly or indirectly 'the store features that attract customers'."

\section{Conclusion}

The results of this research confirm two points. First, the causal relationship was quantitatively clarified through the use of the covariance structural analysis and the degree of relationship between the store kaizen and the store features that attract customers. Second, the proposed methodology has been proved useful in an actual store 
kaizen, based on the comments of the owner of the store investigated. Therefore, the method proposed in this research is confirmed to be useful. The owner managing the store kaizen used the analytical results as a tool to form hypothesis for the store kaizen.

In the future it will be necessary to make good use of the proposed method by applying covariance structural analysis for store kaizen activities. To accomplish this we must focus on two new objectives. One is to produce a guideline on how stores owners should interpret path coefficients in their analytical results. The other is to help store owners anticipate the influences to be expected from specific store kaizen once executed. The store owner in this study suggested this.

\section{References}

1. Taguchi, Y., Tabe, T.: Developing of Decision Support Tool for Deciding Whether to Open a New Shop and How to Improve Existing Shops. In: Abridged Proceedings of the Ninth International Conference on Human-Computer Interaction (HCI' 2001), pp. 251-253 (2001)

2. Shibuya, T., Tabe, T., Taguchi, Y., Maki, N.: An Application of Fuzzy Integral to Measure the Degree of Consumer Satisfaction. In: The Proceedings of the 1st International Conference on Applied Ergonomics (ICAE '96), pp. 1032-1037 (1996)

3. Boollen, K.A.: Structural Equations with Latent Variables. Wiley, Chichester (1989) 